\title{
Idiopathic Juxtafoveal Telangiectasia
}

\section{Jessica Haynes, OD, FAAO}

\section{Jim Williamson, OD, FAAO}

\section{Abstract}

Idiopathic Juxtafoveal Telangiectasia (IJT), also called Idiopathic Macular Telangiectasia, is a group of disorders with varying etiologies that share the common finding of telangiectatic vessels in the juxtafoveal region of the macula. IJT is classically divided into three groups, each with clinically distinct findings and differing etiologies. Type I typically occurs unilaterally, primarily affects males, and is thought to be congenital. Vision loss usually results from macular edema. The most common type, II, normally presents bilaterally, has no sex predilection, and is thought to be acquired. Vision loss occurs not from edema, but from retinal atrophy. The last type, III, is extremely rare and is usually found in association with coexisting systemic or neurological disorders. Vision loss most likely results from retinal ischemia. This paper will describe classifications, clinical findings, etiologies, and treatment options of IJT along with patient case figures.

KEY WORDS:

Idiopathic Juxtafoveal Telangiectasia, Idiopathic Macular Telangiectasia 


\section{INTRODUCTION}

IJT is a condition comprised of three classical divisions, each with different clinical findings, etiologies, and patient demographics. They do share a common feature of capillary abnormalities in the juxtafoveal region that appear on fluorescein angiography as dilated or telangiectatic capillary changes. ${ }^{1}$ IJT can easily be confused for other conditions, and these abnormal capillary findings must be separated from those resultant from other conditions such as diabetes, carotid occlusive disease, retinal vein occlusion, radiation therapy, and others. ${ }^{2}$ The classification also does not include Coat's disease, which has more widespread retinal telangiectatic changes. However, Coat's disease and IJT Type I could be subsets of the same condition that exists as a spectrum of disease. ${ }^{3}$

\section{GASS AND BLODI CLASSIFICATION}

Gass and Owakawa first coined the term IJT in 1982 and initially categorized the condition in four different classes. ${ }^{1}$ Gass and Blodi later revisited the topic in 1993 in a follow up report of the initial work done by Gass and Owakawa at which time they modified the classification into three main categories, each with separate unrelated etiologies and two subsets: Type IA and B, Type IIA and B, and Type IIIA and B. Additionally, Type II was further divided into five stages. ${ }^{3}$ Clinical observations and fluorescein angiography (FA) findings formed the basis of the grouping in this system.

\section{Type I}

Type I IJT presented with easily visible retinal telangiectasia often accompanied with exudation and macular edema, with vision loss resulting from the latter. Gass and Blodi broke down Type I IJT based on the extent of telangiectasia as defined by the number of involved clock hours around the fovea. Type IA had more than two clock hours of telangiectasia while Type IB had less than two clock hours (Figure 1).

Figure 1: Easily visible retinal telangiectasia in a patient with Gass and Blodi Type IA IJT, or Yanuzzi et al.'s Type I aneurysmal telangiectasia.

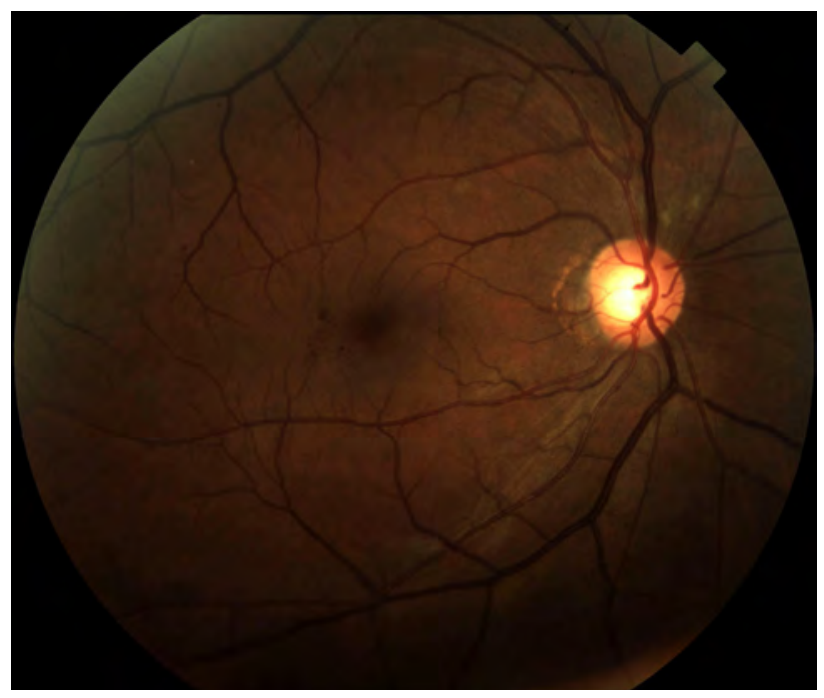

In Gass and Blodi's study, Type IA patients consisted of 31 subjects with a high male predilection (28 male) and a mean patient age of 37 . Entering visual acuity ranged from 20/20 to 20/200. They found Type IA to be unilateral in 30 of the 31 cases. Clinical findings varied between patients, but generally presented with easily visible retinal telangiectasia, macular edema, and exudation. Type IA patients lacked right angle veins, superficial crystalline deposits, intraretinal pigmentary plaques, or subretinal neovascularization (SRNV).

Fluorescein angiography showed quick filling of the irregular telangiectatic vessels around the fovea. These appeared as an irregular round zone of capillary aneurysms and dilated capillaries located temporal to the fovea in $94 \%$ of the cases. They noted minimal capillary occlusion or capillary dropout. Late stage FA revealed intraretinal staining as the abnormal vessels continued to leak. 
Only eight patients in the study displayed the less prevalent Type IB IJT. Again, a high gender predilection existed with seven of the patients being male. The average age was 42 . All but two eyes (20/25 and 20/30) exhibited 20/20 visual acuity.

Findings were again unilateral in most cases (seven out of eight) but were confined to two clock hours or less of still easily visible telangiectasia. A few flecks of exudates occurred in most cases, but less than that seen with Type IA. Fluorescein angiography showed early filling of one to four capillary aneurysms and of the irregular capillaries followed by late staining around the rare aneurysms.

\section{Type II}

Unlike Type I, Type II IJT rarely displays macular edema and exudation. In these patients, outer retina atrophy is the main cause of vision loss. Gass and Blodi classified Type II into subsets A and B based on the age of presentation.

Type IIA consisted of 92 patients with equal gender incidence and an average age of 55 years. Entering visual acuity ranged from 20/15 to hand motion. Presentation was bilateral, though often asymmetric, in 90 out of 92 patients. Unlike Type I, these patients exhibited either minimal, poorly visible, or no clinically discernible telangiectasia. These abnormal capillaries would light up early with FA and then gradually stain in late stages (Figure 2). All cases involved the temporal parafovea. Additional clinical findings of Type IIA included right angle veins, pigmented retinal plaques, superficial crystalline deposits, and SRNV (Figures 3 and 4). Gass and Blodi also described what appeared to be foveal atrophy on funduscopic evaluation, similar to that of a lamellar macular hole (Figure 4).

Figure 2: Early phase FA (left) shows the classic finding of punctate hyperfluorescence temporal to the fovea and the atypical location of more areas superior to the macula. Late phase reveals increased hyperfluorescence (right).
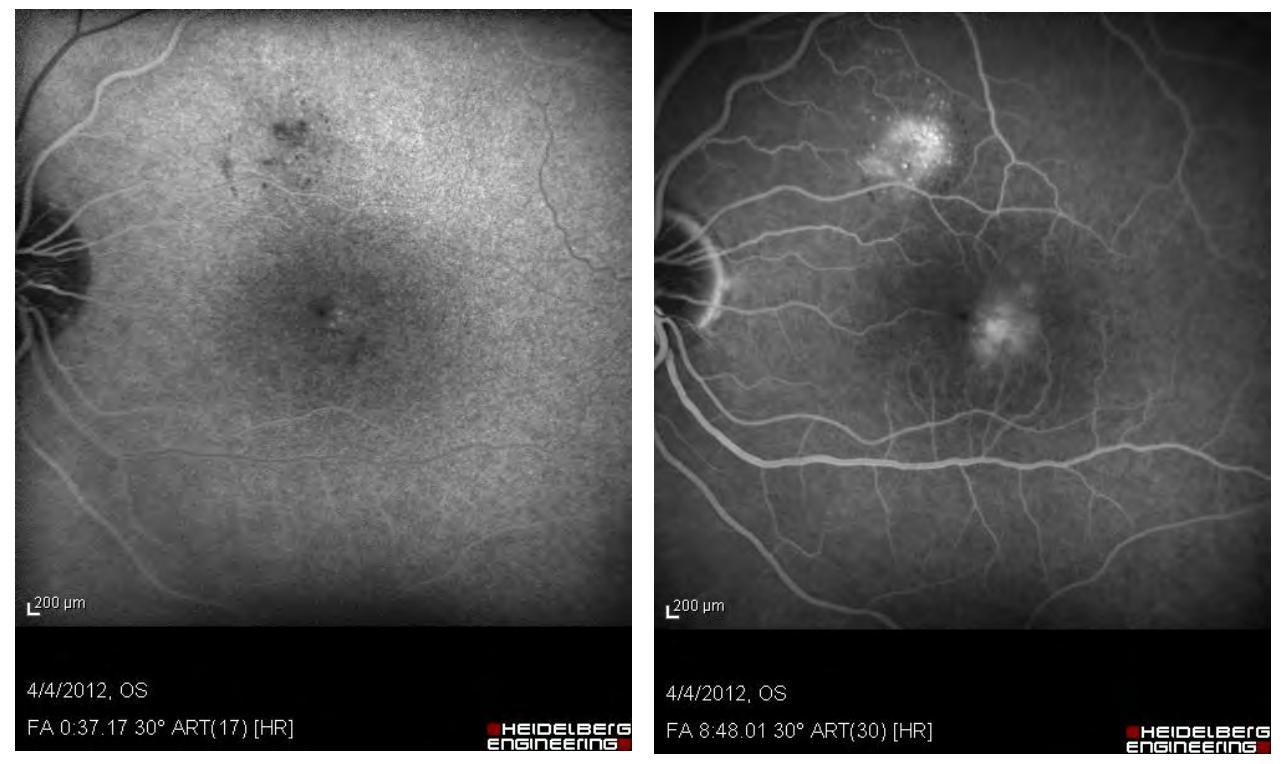

Gass and Blodi developed five stages of Type IIA. In their findings, visual acuity remained unaffected in Stages 1 and 2. Patients typically became symptomatic in Stage 3, and Stage 5 resulted in poor visual acuity.

- Stage 1: Biomicroscopically normal fundus, minimal or no capillary abnormality in early FA, mild staining in late FA

- Stage 2: Slight graying of perifoveal retina, minimal or no visible telangiectasia, capillary telangiectasia in early FA

- Stage 3: Right angle veins

- Stage 4: Pigmented retinal plaques

- Stage 5: Subretinal neovascularization 
Figure 3: Gass and Blodi Type IIA Stage 4 (note the prominent right angle vein stretching from the superior temporal venous branch directly to the pigment plaque), or Yanuzzi et al.'s Type II perifoveal telangiectasia, non-proliferative stage.

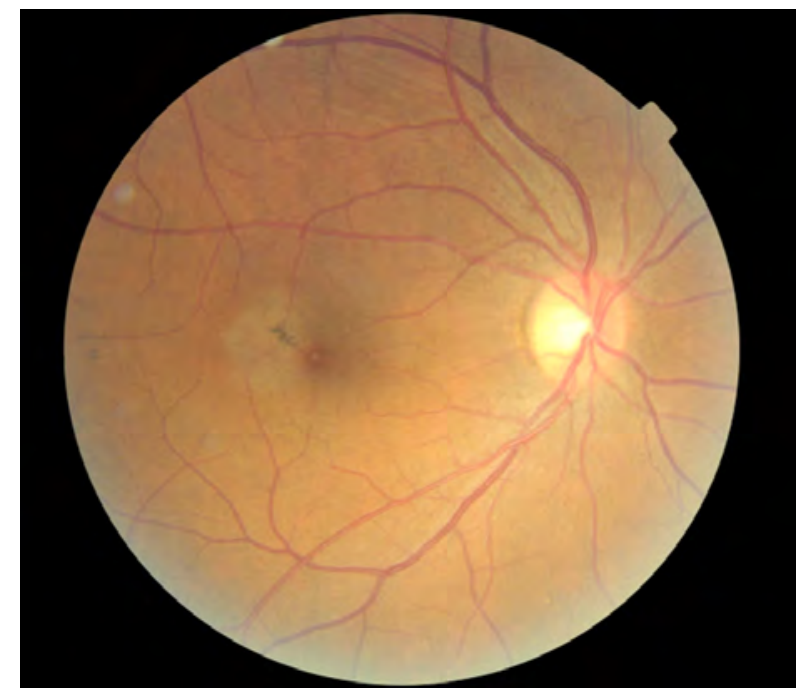

Figure 4: Type II IJT with crystalline deposits, perifoveal retinal whitening, and a foveal appearance similar to that of a lamellar macular hole.

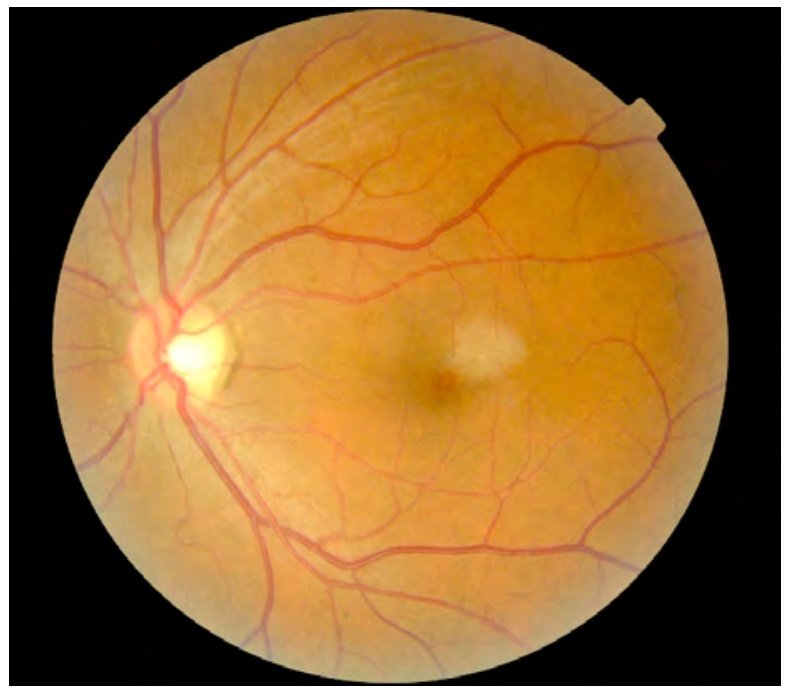

Type IIB consisted of only two patients who were brothers aged nine and 12. Both presented with bilateral SRNV and subtle juxtafoveal telangiectasia, but without right angle veins, crystalline deposits, or pigmented plaques.

\section{Type III}

Occlusive capillary dropout along with retinal telangiectasia characterized Type III. All had minimal exudation and edema. Type IIIA consisted of three women aged 41 to 59, all with bilateral findings. Their visual acuity ranged from $20 / 20$ to $20 / 50$. One reported polycythemia vera, another gout, and a third hyperglycemia. Type IIIB consisted of five men aged 35 to 41 all with CNS disorders. Two of them were brothers. 
YANUZZI ET AL. CLASSIFICATION

In 2006, Yanuzzi et al. proposed a simpler classification based on clinical, FA, and optical coherence tomography (OCT) findings in patients with IJT. Again there were three main categories, but without subsets in each main division. They referred to Type I as "aneurysmal telangiectasia", Type II as "perifoveal telangiectasia”, and Type III as "occlusive telangiectasia”. Similar to Gass and Blodi, Yanuzzi et al. further divided Type II into stages, but only two instead of five: Non-proliferative and proliferative. ${ }^{4}$

\section{Type I - Aneurysmal Telangiectasia}

Yanuzzi et al. reclassified Type IA and IB to "aneurysmal telangiectasia.” In this study, those with less than two clock hours of telangiectasia eventually progressed to having more extensive involvement. Therefore, they felt that Type IB was just an earlier presentation of Type IA (Figure 1).

There were 10 patients (nine men, one female) in the report with aneurysmal telangiectasia with an average age of 56. All but the female patient displayed unilateral findings consistent with those reported by Gass and Blodi, such as easily visible telangiectasia, exudation, and macular edema. OCT confirmed the presence of macular edema. Once again there were no findings of neovascularization, pigment proliferation, or crystalline deposits, and there was minimal ischemia.

\section{Type II - Perifoveal Telangiectasia}

"Perifoveal telangiectasia" replaced both Type IIA and Type IIB. Yanuzzi et al. believed Type IIB to be a familial abnormality as there had been no other reported cases in the literature. They also found that not all patients went through each stage of Type II previously described by Gass and Blodi, and so they categorized Type II into proliferative and non-proliferative based on the presence or absence of SRNV.

Perifoveal telangiectasia patients in this study consisted of 11 males and 15 females with an average age of 59. Clinical and FA findings were similarly consistent with those seen by Gass and Blodi (Figure 2). All cases were bilateral, but again with some asymmetry. Early signs included mild loss of retinal transparency and mild perifoveal retinal whitening without obvious telangiectasia which appeared later in the disease. Also seen in some were pigment plaques, crystalline deposits, and right angle vessels which Yanuzzi et al. explained could be arteriolar or venular in origin (Figures 3 and 4).

Optical coherence tomography findings provided enlightening insight into perifoveal telangiectasia. Even though the FA showed late staining, OCT confirmed the absence of intraretinal edema. Cystic retinal spaces often bordered anteriorly by the internal limiting membrane (ILM) were present, but they were not secondary to edematous leakage. Instead, they resulted from retinal atrophy. Yanuzzi et al. called these spaces inner lamellar cysts with ILM drape (Figure 5). Optical coherence tomography also revealed a progressive loss of the outer retina along with photoreceptor atrophy (Figure 6). The level of atrophy correlated with the patients' visual acuities. Pigmentary plaques appeared as nonspecific subretinal reflectance with posterior shadowing. The crystalline deposits were too small to image with OCT. In the event of SRNV, resultant macular edema, hemorrhage, macular detachment, or fibrosis could also be visualized with OCT.

Due to the apparent features of Type II IJT seen with OCT imaging, some suggest new grading criteria based on OCT findings. ${ }^{5,6}$ In addition to Yanuzzi et al.'s OCT findings, additional reports describe early retinal changes using macular pigment optical density, confocal scanning laser ophthalmoscope, and fundus autofluorescence (FAF). ${ }^{7}$ According to some, FAF may detect the earliest signs of Type II IJT due to macular pigment breakdown which allows increased autofluorescence to show through from the underlying retinal pigment epithelium (RPE). ${ }^{8,9}$ It eventually affects the RPE itself and causes an increase in both lipofuscin and subsequent autofluorescence (Figure 7). Further release of disrupted RPE pigment causes a blocking effect and leads to a mottled appearance of both hyper- and hypo-fluorescence. 8,9

\section{Type III - Occlusive Telangiectasia}

Yanuzzi et al. did not include any Type III or occlusive telangiectasia cases in their report. They believed it to be primarily an ischemic foveal disease with compensatory changes in the capillary bed, and argued that it be omitted from the macular telangiectasia classification as it is an ocular manifestation of systemic or cerebral familial disease. 


\section{PROPOSED ETIOLOGIES}

Type I IJT is thought to be a congenital malformation of the retinal vasculature which allows for leakage of fluid and exudation from the capillary vessels. ${ }^{3,4}$ As mentioned previously, edema is the main cause of vision loss in these patients. Yanuzzi et al. and Gass and Blodi both considered that Type I IJT or aneurysmal telangiectasia could be a more focal variant of Coat's disease and that perhaps they exist on a spectrum of diseases caused by congenital retinal telangiectasia formation.

Figure 5: SD-OCT of an intraretinal cyst with overlying ILM drape and underlying photoreceptor damage.

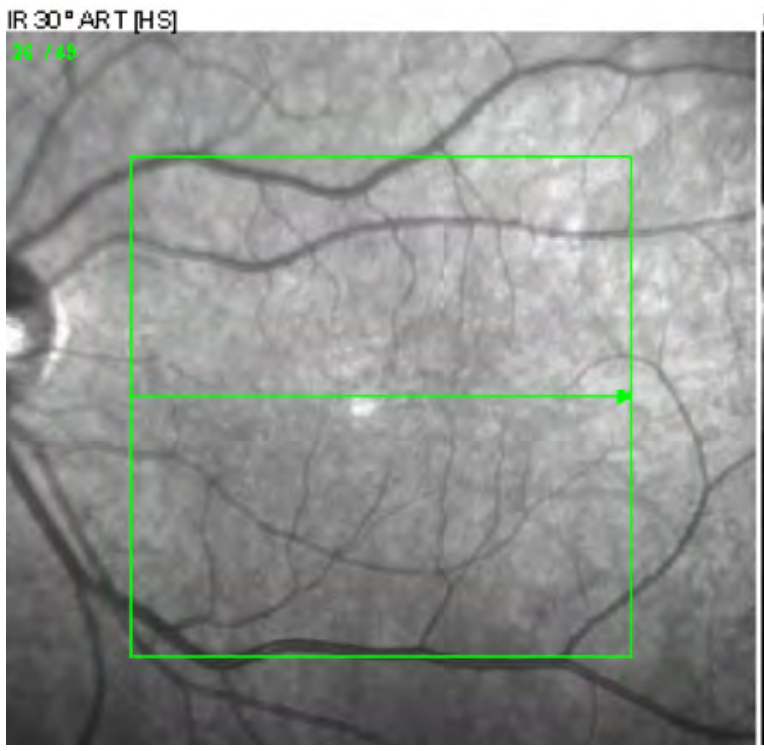

OCT $20^{\circ}(60 \mathrm{~mm}) \mathrm{ART}(19) \mathrm{Q}: 28 \mathrm{HS}$

Figure 6: $S D$-OCT reveals outer retinal atrophy.

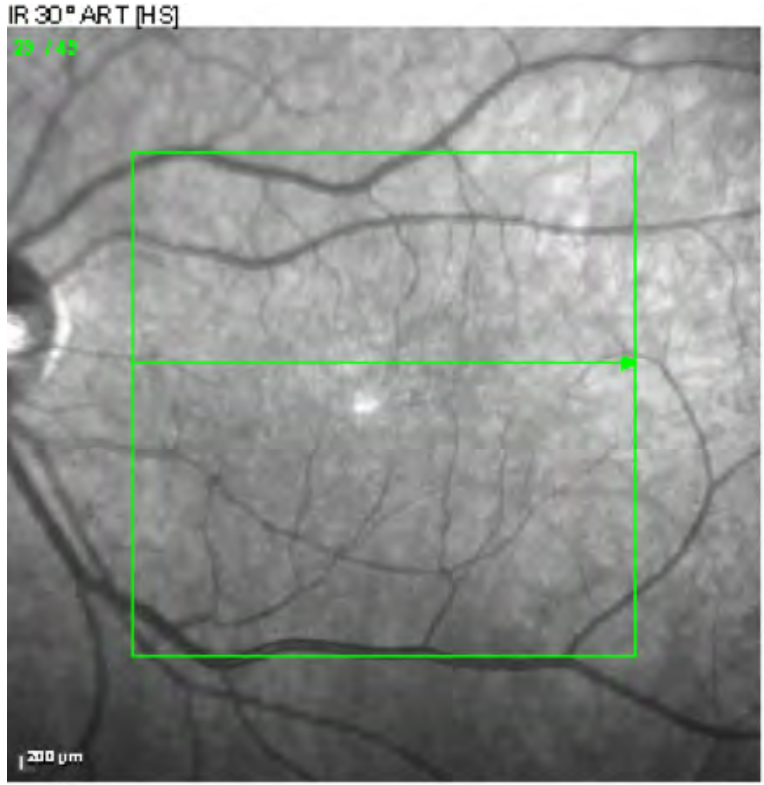

OCT $20^{\circ}(6,0 \mathrm{~mm}) \operatorname{ART}(16)$ Q:27 HS

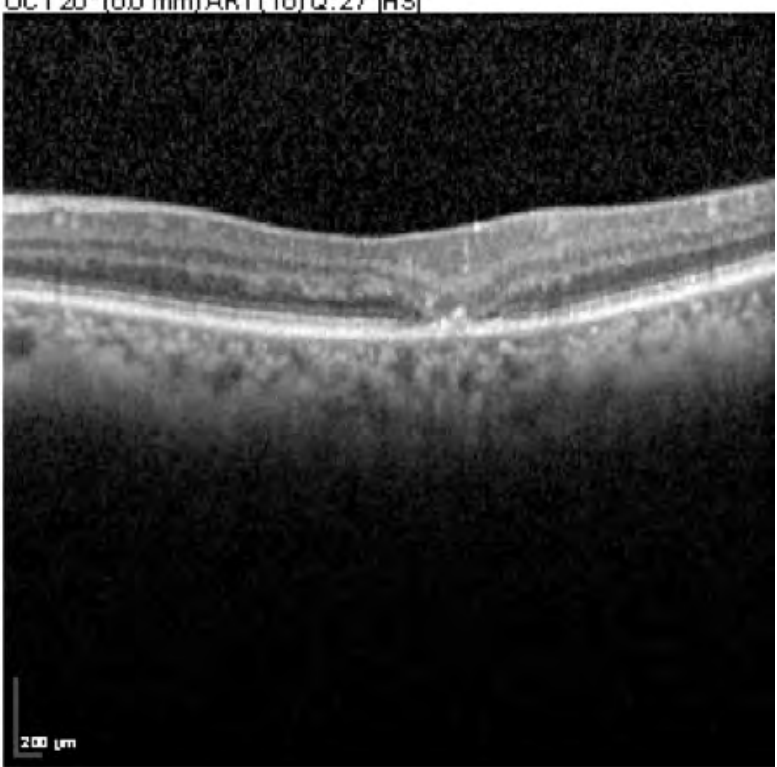


Type II IJT may be an acquired condition in which the malformations of the retinal vasculature present later in life. ${ }^{3,4}$ A histological study performed by Green et al. on a patient with Type II IJT showed narrowing of the vessel lumen versus the telangiectatic or dilated appearance noted clinically and on FA. Increased width of the basement membrane along with degeneration of pericytes and endothelial cells led to thickening of the retinal capillaries. The authors conceived that breaks in the endothelium allowed fluorescein to leak into the thickened capillary walls which accounted for the late staining seen on FA. This report also found that capillary proliferation extended into the outer retinal layers down to the photoreceptors..$^{10}$ A different histopathological report from a more advanced case of Type II IJT with SRNV did show dilation of retinal capillaries that reached into the outer retinal layers and subretinal space. Pigment migration also occurred along the course of the proliferating vessels. ${ }^{11}$

Figure 7: FAF details an area of central hypofluorescence corresponding to the pigment plaque surrounded by a hyperfluorescent ring indicating tissue degradation.

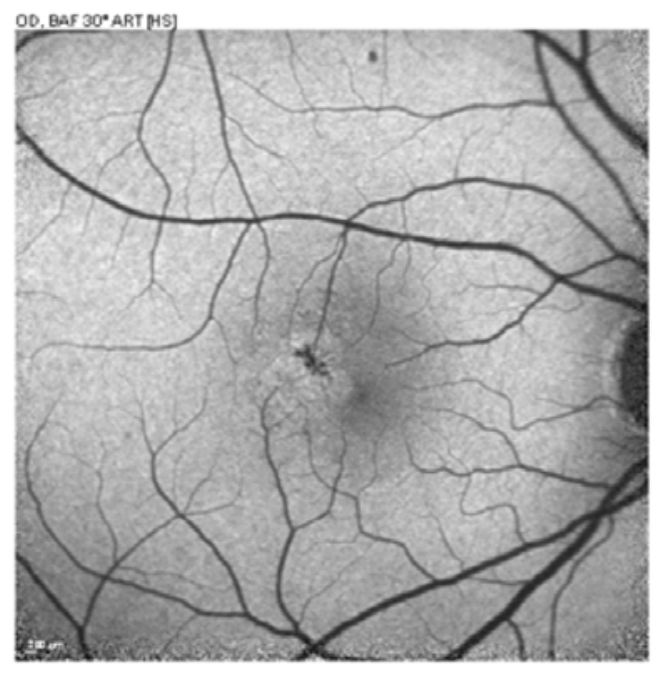

Speculation exists on whether the capillary abnormalities are the initial step in a process that leads to nutritional and metabolic damage to the retina, or if there is some sort of retinal change that precedes and leads to the formation of the abnormal capillaries. Müller cells garner the most suspicion due to their importance in maintaining proper health of the vasculature endothelium and sensory retina. ${ }^{12,13}$ A primary dysfunction in these cells may lead to capillary abnormalities and retinal atrophy, with the superficial crystalline deposits representing remnants of degenerated Müller cells. ${ }^{14}$

SRNV formation appears to stem from proliferation and anastomoses that exist within the blood vessels of the retina. This differs from the retino-choroidal anastomoses found in macular degeneration. The reason for retinal capillary proliferation is unclear, but photoreceptor atrophy may give the capillaries easier access to the subretinal space..$^{15}$

\section{TREATMENT OPTIONS}

Treatments for Type I IJT, or aneurysmal telangiectasia, aim at the destruction of the abnormal capillaries and aneurysms with photocoagulation or photodynamic therapy (PDT); or the stabilization of the blood retinal barrier with injectable medications. Limited options exist for those with Type II IJT due to the non-edematous nature of the disease, unless SRNV forms. Then treatments include laser photocoagulation, PDT, intravitreal triamcinolone injections, and intravitreal anti VEG-F agents.

\section{Photocoagulation}

Photocoagulation benefitted only those with Type I IJT, as noted by both Gass and Owakawa; and Gass and Blodi in their respective reports. They found a potential improvement in vision and exudation with photocoagulation. The procedure proved to be non-beneficial in those with Type II IJT. ${ }^{3,16}$ 


\section{Photodynamic Therapy}

Photodynamic therapy has also shown potential for improvement in vision, exudation, and edema in those with Type I IJT. ${ }^{17}$ The benefits in Type II IJT, however, are limited to those with SRNV. Several reports show decreased leakage on FA as well as potential improvement or stabilization in vision in those with Type II with SRNV treated with PDT. ${ }^{18,19,20,21}$ Even though there is no frank visual improvement in many of these cases, it is important to remember that non-treated SRNV has very poor visual prognosis with $80 \%$ having final visual acuities of worse than 20/200.22 In those with Type II IJT without SRNV, treatment with PDT temporarily improved leakage on FA, but did not improve visual acuity. ${ }^{23}$

\section{Intravitreal Injections}

Injection of intravitreal triamcinolone decreases leakage in Type I IJT and Type II IJT both with and without SRNV. Some report improvement in both macular edema as shown with OCT and in vision in those with Types I and II IJT with SRNV. However, one must consider the high side effect profile with steroid injections. Additionally, even though leakage improves on FA in Type II IJT without SRNV, there is no improvement in retinal thickness with OCT or visual acuity in these cases. The effects of treatment in those who do show improvement are short lived with recurrent fluid leakage in three to six months. ${ }^{24,25}$

Similar to intravitreal triamcinolone, injection of anti VEG-F agents decrease FA leakage in Types I and II IJT with and without SRNV with a much more favorable side effect profile. . $6,27,28,29,3,3,31$ Reports indicate improvement in macular edema, retinal thickness, and vision in Types I and II IJT with SRNV. In Type II IJT patients without SRNV, few cases recorded improvement in retinal thickness and visual acuity; however, the majority failed to note a benefit in those with Type II IJT without SRNV. Although treatment with anti VEG-F has not been consistently shown to improve vision in those with Type II without SRNV, it is postulated that since it does reduce leakage from the abnormal blood vessels, that long-term therapy could potentially slow retinal atrophy. However, VEG-F is also needed in a certain level to maintain retinal vasculature health, and it is possible that long-term therapy could actually exacerbate retinal cell death..$^{32}$

Due to the rare incidences of these conditions, most treatment studies available include a minimal number of patients or are even single case reports. Long-term follow up data is limited as well. Since there is such a poor untreated prognosis in those with SRNV in Type II, it is important to remember that stabilization of vision even without improvement could be a success in treatment. Additionally, substances that could slow the neurodegenerative process that appears to be taking place in Type II IJT are currently being investigated. One particular molecule, called ciliary neurotrophic growth factor, slows photoreceptor cell death in animal models and recently demonstrated safety in phase 1 clinical trials in humans. ${ }^{33} \mathrm{At}$ this time, however, treatment is reserved for those with SRNV in Type II IJT.

\section{DIFFERENTIAL DIAGNOSES}

Differential diagnosis for IJT should include other identifiable causes of macular telangiectasia such as diabetic retinopathy, retinal vein occlusion, carotid occlusive disease, radiation retinopathy, and others. Careful case history of potential systemic etiologies of retinal telangiectasia should be thoroughly considered. Diabetic retinopathy is likely the most commonly misattributed diagnosis for those with Type I IJT; however, the presence of fairly isolated, mostly temporal, unilateral retinal telangiectasia or hemorrhaging should raise a red flag for the diagnosis of Type I IJT.

Type II IJT is less commonly confused with other causes of retinal telangiectasia as it most often presents with one or more of its classic findings of crystalline deposits, retinal pigment plaques, right angle veins, and outer retinal atrophy. Due to the profound funduscopic appearance of the pigmented plaques and significant outer retinal atrophy on OCT, this condition can be confused with conditions such as age related macular degeneration (AMD), chronic or recurrent central serous retinopathy, toxic retinopathies from medications such as hydroxychloroquine, or retinal scarring from conditions such as presumed ocular histoplasmosis (POHS). Differentials in the formation of SRNV should include other conditions with a similar finding, such as wet AMD, POHS, polypoidal choroidal vasculopathy, and others.

\section{VISUAL PROGNOSIS}

Visual prognosis for IJT varies case to case. As discussed previously, vision loss in Type I IJT is related, at least initially, to the level of macular edema present, which can potentially show improvement with various treatment 
modalities. However, due to the rarity of the condition, little information exists to determine long-term prognosis. The condition is a chronic one, and does require continued monitoring and possibly long-term treatment.

Vision loss from Type II IJT develops due to atrophy of the outer retina and photoreceptors. Due to the location of retinal atrophy being temporal to the foveal center, visual acuity often remains good in these patients until the development of SRNV. Data from the MacTel Study group showed that $42 \%$ of all patients had visual acuity of $20 / 25$ or better..$^{34}$ However, this photoreceptor damage can create paracentral scotomas that lead to patient symptoms even with good retained visual acuity. ${ }^{35}$ Various reports demonstrate that even with acceptable visual acuity, patients become symptomatic for patient perceived metamorphopsia, decreased reading speed, and difficulty in reading - the latter of which being the most frequently reported initial symptom. ${ }^{36,37}$ These symptoms arise most commonly between the ages of 50 and 69. Exact values for long-term prognosis for Type II IJT varies between reports but patients do have increased risk for both decreased visual acuity as well as decreased quality of life from vision loss. ${ }^{38,39}$

\section{CONCLUSION}

Diagnosis of IJT can be complicated by clinical appearances mimicking other conditions. For example Type I IJT can easily be confused with diabetic retinal changes. While treatment options for the complication of macular edema from Type I IJT are essentially the same as those for diabetic macular edema, it is still important to make the correct diagnosis. While systemic conditions should still be considered and ruled out, a patient with Type I IJT could have unwarranted psychological distress if told they have retinal complications from diabetes when they don't have the disease.

Also, even though there is currently no known beneficial treatment in the case of Type II IJT without SRNV, it is still important to make an accurate assessment in order to educate patients properly about their condition and prognosis. Patients should be made aware that the condition is a progressive retinal disease which can lead to central vision loss, metamorphopsia, and paracentral scotomas. They also need to be thoroughly educated on how to properly monitor their vision monocularly, and to be examined periodically for SRNV. With proper understanding of IJT, optometrists can monitor these conditions appropriately, referring for additional treatment when necessary. $\bullet$

Table: Comparison of the Two IJT Classification Schemes

\begin{tabular}{|l|l|l|l|l|l|l|}
\cline { 2 - 7 } \multicolumn{2}{c|}{} & \multicolumn{2}{|l}{ Type I IJT } & \multicolumn{2}{l}{ Type II IJT } & \multicolumn{2}{l|}{ Type III IJT } \\
\cline { 2 - 7 } & Gass and Blodi & Yanuzzi et al. & Gass and Blodi & Yanuzzi et al. & Gass and Blodi & Yanuzzi et al. \\
\hline Subtypes & IA and IB & None & IIA and IIB & None & IIIA and IIIB & N/A \\
\hline $\begin{array}{l}\text { Gender } \\
\text { prevalence }\end{array}$ & Male & Male & $\begin{array}{l}\text { IIA - None } \\
\text { IIB - Brothers }\end{array}$ & None & $\begin{array}{l}\text { IIIA - female } \\
\text { IIIB - male }\end{array}$ & N/A \\
\hline Laterality & Unilateral & Unilateral & Bilateral & Bilateral & Bilateral & N/A \\
\hline Stages & No & No & IIA - yes (five) & Yes (two) & No & N/A \\
\hline $\begin{array}{l}\text { Clinical } \\
\text { findings }\end{array}$ & $\begin{array}{l}\text { Both - easily } \\
\text { visible retinal } \\
\text { telangiectasia, } \\
\text { exudation, } \\
\text { macular edema } \\
\text { IA two } \\
\text { clock hours of } \\
\text { involvement } \\
\text { IB }<\text { two } \\
\text { clock hours of } \\
\text { involvement }\end{array}$ & $\begin{array}{l}\text { Easily visible } \\
\text { relangiectasia, } \\
\text { exudation, } \\
\text { macular edema }\end{array}$ & $\begin{array}{l}\text { Both - } \\
\text { minimally } \\
\text { visible to no } \\
\text { observable } \\
\text { telangectiasia } \\
\text { IA - Right } \\
\text { angle veins, } \\
\text { pigmented } \\
\text { retinal plaques, } \\
\text { superficial } \\
\text { crystalline } \\
\text { deposits, SRNV }\end{array}$ & $\begin{array}{l}\text { Mild loss } \\
\text { of retinal } \\
\text { transperancy } \\
\text { without } \\
\text { obvious } \\
\text { telangiectasia, } \\
\text { pigment } \\
\text { plaques, } \\
\text { crystalline } \\
\text { deposits, right } \\
\text { angle vessels, } \\
\text { SRNV }\end{array}$ & $\begin{array}{l}\text { Occlusive } \\
\text { capillary } \\
\text { dropout, } \\
\text { minimal } \\
\text { exudation and } \\
\text { edema } \\
\text { IIIB - } \\
\text { associated CNS } \\
\text { disorders }\end{array}$ & N/A \\
\hline
\end{tabular}




\section{References}

1. Gass, J. D., and R. T. Owakawa. "Idiopathic Juxtafoveolar Retinal Telangiectasis." Archives of Ophthalmology 100.5 (1982): 769-80.

2. Issa, Peter Charbel, Mark C. Gillies, Emily Y. Chew, Alan C. Bird, Tjebo F. C. Heeren, Tunde Peto, Frank G. Holz, and Hendrik P. N. Scholl. "Macular Telangiectasia Type 2." Prog Retin Eye Res 34 (2013): 49-77.

3. Gass, J. Donald M., and Barbara A. Blodi. "Idiopathic Juxtafoveolar Retinal Telangiectasis.” Ophthalmology 100.10 (1993): 1536-546.

4. Yannuzzi, Lawrence A. "Idiopathic Macular Telangiectasia." Archives of Ophthalmology Arch Ophthalmol 124.4 (2006): 450. Web.

5. Cohen, Steven M., Mark L. Cohen, Fayssal El-Jabali, and Scott E. Pautler. "Optical Coherence Tomography Findings In Nonproliferative Group 2A Idiopathic Juxtafoveal Retinal Telangiectasis.” Retina 27.1 (2007): 59-66

6. Sanchez JG, Garcia RA, Wu L, Berrocal MH, Graue-Wiechers F, Rodriguez FJ, et al. "Optical coherence tomography characteristics of group 2A idiopathic parafoveal telangiectasis." Retina 27 (2007): 1214-20.

7. Helb, Hans-Martin, Peter Charbel Issa, Rob L. P. Van Der Veen, Tos T. J. M. Berendschot, Hendrik P. N. Scholl, and Frank G. Holz. "Abnormal Macular Pigment Distribution In Type 2 Idiopathic Macular Telangiectasia." Retina 28.6 (2008): 808-16.

8. Bottoni F, Eandi CM, Pedenovi S, Staurenghi G. "Integrated clinical evaluation of Type $2 \mathrm{~A}$ idiopathic juxtafoveolar retinal telangiectasis." Retina 30 (2010): 317-26.

9. Wong WT, Forooghian F, Majumdar Z, Bonner RF, Cunningham D, Chew EY. "Fundus autofluorescence in type 2 idiopathic macular telangiectasia: Correlation with optical coherence tomography and microperimetry." Am J Ophthalmol 148 (2009): 573-83.

10. Green, W. Richard, H. A. Quigley, Z. De La Cruz, and Bruce Cohen. "Parafoveal Retinal Telangiectasis." Retina 25.Supplement (2005): $162-70$.

11. Eliassi-Rad, Babak, and W. Richard Green. "Histopathologic Study Of Presumed Parafoveal Telangiectasis." Retina 19.4 (1999): 332.

12. Powner, Michael B., Mark C. Gillies, Marina Tretiach, Andrew Scott, Robyn H. Guymer, Gregory S. Hageman, and Marcus Fruttiger. "Perifoveal Muller Cell Depletion in a Case of Macular Telangiectasia Type 2." Ophthalmology 117.12 (2010): 2407-416.

13. Gass, JD. "Müller cell cone, an overlooked part of the anatomy of the fovea centralis: Hypotheses concerning its role in the pathogenesis of macular hole and foveomacualr retinoschisis." Arch Ophthalmol 117 (1999) 821-823.

14. Wu, Lihteh. "Multimodality Imaging in Macular Telangiectasia 2: A Clue to Its Pathogenesis." Indian J Ophthalmol Indian Journal of Ophthalmology 63.5 (2015): 394.

15. Gass JD. "Chorioretinal anastomosis probably occurs infrequently in type $2 \mathrm{~A}$ idiopathic juxtafoveolar retinal telangiectasis." Arch Ophthalmol 121 (2003): 1345-6.

16. Park, Donald W., Howard Schatz, H. Richard Mcdonald, and Robert N. Johnson. "Grid Laser Photocoagulation for Macular Edema in Bilateral Juxtafoveal Telangiectasis.” Ophthalmology 104.11 (1997): 1838-846.

17. Kotoula, Maria G., Dimitrios Z. Chatzoulis, Constantinos H. Karabatsas, Aristoteles Tsiloulis, and Evangelia E. Tsironi. "Resolution of Macular Edema in Idiopathic Juxtafoveal Telangiectasis Using PDT." Ophthalmic Surg Lasers Imaging Ophthalmic Surgery, Lasers, and Imaging 40.1 (2009): 65-67.

18. Snyers, Bernadette, Claire Verougstraete, Laurence Postelmans, Anita Leys, and Philip Hykin. "Photodynamic Therapy of Subfoveal Neovascular Membrane in Type 2A Idiopathic Juxtafoveolar Retinal Telangiectasis." American Journal of Ophthalmology 137.5 (2004): 812-19.

19. Smithen, Lindsay M., and Richard F. Spaide. "Photodynamic Therapy and Intravitreal Triamcinolone for a Subretinal Neovascularization in Bilateral Idiopathic Juxtafoveal Telangiectasis.” American Journal of Ophthalmology 138.5 (2004): 884-85.

20. Lee, Bailey L. "Bilateral Subretinal Neovascular Membrane In Idiopathic Juxtafoveolar Telangiectasis." Retina 16.4 (1996): 344-45.

21. Potter, Michael J., Shelagh M. Szabo, Eleanor Y. Chan, and Andrew H.c Morris. "Photodynamic Therapy of a Subretinal Neovascular Membrane in Type 2A Idiopathic Juxtafoveolar Retinal Telangiectasis." American Journal of Ophthalmology 133.1 (2002): 149-51.

22. Engelbrecht, Nicholas E. "Neovascular Membranes Associated With Idiopathic Juxtafoveolar Telangiectasis." Archives of Ophthalmology Arch Ophthalmol 120.3 (2002): 320.
23. Lahitte, Ghislaine Ducos De, Salomon Y. Cohen, and Alain Gaudric "Lack of Apparent Short-term Benefit of Photodynamic Therapy in Bilateral, Acquired, Parafoveal Telangiectasis without Subretinal Neovascularization.” American Journal of Ophthalmology 138.5 (2004): 892-94.

24. Alldredge, Claron D., and Bruce R. Garretson. "Intravitreal Triamcinolone For The Treatment Of Idiopathic Juxtafoveal Telangiectasis." Retina 23.1 (2003): 113-16.

25. Wu, Lihteh, Teodoro Evans, J. Fernando Arévalo, María H. Berrocal, Francisco J. Rodríguez, María Hsu, and Juan G. Sánchez. "Long-Term Effect Of Intravitreal Triamcinolone In The Nonproliferative Stage Of Type Ii Idiopathic Parafoveal Telangiectasia." Retina 28.2 (2008): 314-19.

26. Kovach, Jaclyn L., and Philip J. Rosenfeld. "Bevacizumab (Avastin) Therapy For Idiopathic Macular Telangiectasia Type Ii.” Retina 29.1 (2009): 27-32.

27. Júnior, Otacílio Oliveira Maia, Maria Teresa Brizzi Chizzotti Bonanomi, Walter Yukihiko Takahashi, Vinícius Paganini Nascimento, and Beatriz Sayuri Takahashi. "Intravitreal Bevacizumab for Foveal Detachment in Idiopathic Perifoveal Telangiectasia." American Journal of Ophthalmology 144.2 (2007): 296-99.

28. Issa, P. C., R. P. Finger, F. G. Holz, and H. P N Scholl. "Eighteenmonth Follow-up of Intravitreal Bevacizumab in Type 2 Idiopathic Macular Telangiectasia." British Journal of Ophthalmology 92.7 (2008): 941-45.

29. Gamulescu, Maria-Andreea, Andreas Walter, Helmut Sachs, and Horst Helbig. "Bevacizumab in the Treatment of Idiopathic Macular Telangiectasia." Graefes Arch Clin Exp Ophthalmol Graefe's Archive for Clinical and Experimental Ophthalmology 246.8 (2008): 1189-193.

30. García-Ben, A., F. Gómez-Ulla, and M.j. Rodriguez-Cid. "Intravitreal Bevacizumab in the Treatment of Idiopathic Juxtafoveal Telangiectasis Type I." Archivos De La Sociedad Española De Oftalmología (English Edition) 89.7 (2014): 269-71.

31. Nakhwa, Chinmayp, and Manavid Sindal. "Idiopathic Macular Telangiectasia Type 1 with Ruptured Retinal Arterial Macroaneurysm Post Intravitreal Bevacizumab." Middle East African Journal of Ophthalmology Middle East Afr J Ophthalmol 22.3 (2015): 396.

32.) Kupitz, Elke H., Tjebo F. C. Heeren, Frank G. Holz, and Peter Charbel Issa. "Poor Long-Term Outcome Of Anti-Vascular Endothelial Growth Factor Therapy In Nonproliferative Macular Telangiectasia Type 2." Retina 35.12 (2015): 2619-626.

33. Chew, Emily Y., Traci E. Clemons, Tunde Peto, Ferenc B. Sallo, Avner Ingerman, Weng Tao, Lawrence Singerman, Steven D. Schwartz, Neal S. Peachey, and Alan C. Bird. "Ciliary Neurotrophic Factor for Macular Telangiectasia Type 2: Results From a Phase 1 Safety Trial." American Journal of Ophthalmology 159.4 (2015): 659-666.

34. Clemons, Traci E., Marc C. Gillies, Emily Y. Chew, Alan C. Bird, Tunde Peto, Maria J. Figueroa, and Molly W. Harrington. "Baseline Characteristics of Participants in the Natural History Study of Macular Telangiectasia (MacTel) MacTel Project Report No. 2." Ophthalmic Epidemiology 17.1 (2010): 66-73.

35. Heeren, Tjebo F. C., Traci Clemons, Hendrik P. N. Scholl, Alan C. Bird, Frank G. Holz, and Peter Charbel Issa. "Progression of Vision Loss in Macular Telangiectasia Type 2." Investigative Opthalmology \&t Visual Science Invest. Ophthalmol. Vis. Sci. 56.6 (2015): 3905.

36. Heeren, Tjebo F. C., Frank G. Holz, and Peter Charbel Issa. "First Symptoms And Their Age Of Onset In Macular Telangiectasia Type 2." Retina 34.5 (2014): 916-19.

37. Finger, Robert P., Peter Charbel Issa, Rolf Fimmers, Frank G. Holz, Gary S. Rubin, and Hendrik P. N. Scholl. "Reading Performance Is Reduced by Parafoveal Scotomas in Patients with Macular Telangiectasia Type 2." Investigative Opthalmology \& Visual Science Invest. Ophthalmol. Vis. Sci. 50.3 (2009): 1366.

38. Watzke, Robert C., Michael L. Klein, James C. Folk, Samuel G. Farmer, Richard S. Munsen, Robert J. Champfer, and K. R. Sletten. "Long-Term Juxtafoveal Retinal Telangiectasia.” Retina 25.6 (2005): 727-35.

39. Lamoureux, Ecosse L., Rebecca M. Maxwell, Manjula Marella, Mohamed Dirani, Eva Fenwick, and Robyn H. Guymer. "The Longitudinal Impact of Macular Telangiectasia (MacTel) Type 2 on Vision-Related Quality of Life." Investigative Opthalmology \&t Visual Science Invest. Ophthalmol. Vis. Sci. 52.5 (2011): 2520. 